\title{
Downregulation of phosphoglycerate kinase 1 by shRNA sensitizes U251 xenografts to radiotherapy
}

\author{
YI-JUN CHENG ${ }^{1}$, HAO DING $^{1}$, HUA-QING DU ${ }^{2}$, HUA YAN $^{1}$, JIN-BING ZHAO ${ }^{1}$, \\ WEN-BIN ZHANG ${ }^{1}$, YUAN-JIE ZOU ${ }^{1}$, HONG-YI LIU ${ }^{1}$ and HONG XIAO ${ }^{2}$ \\ ${ }^{1}$ Department of Neurosurgery and ${ }^{2}$ Neuro-Psychiatric Institute, Nanjing Brain Hospital \\ Affiliated to Nanjing Medical University, Nanjing, Jiangsu, P.R. China
}

Received April 6, 2014; Accepted July 9, 2014

DOI: $10.3892 /$ or.2014.3353

\begin{abstract}
Phosphoglycerate kinase 1 (PGK1) has been demonstrated to be involved in radioresistance. The present study was designed to investigate the effect of PGK1 on the radioresistance in vivo. $\mathrm{U} 251$ glioma cells were transfected with the short hairpin RNA (shRNA)-PGK1 and pcDNA3.1-PGK1 using Lipofectamine 2000. The radiosensitivity of U251 xenografts was observed by tumor growth curve following radiotherapy. Quantitative PCR, western blot analysis and immunohistochemistry were performed to evaluate PGK1 expression in the xenografts from the different tumor models. The expression of PGK1 was maximally inhibited in response to shRNA4 at $24 \mathrm{~h}$ after the transfection in vitro. Tumor growth of the U251 xenografts was significantly inhibited following treatment with shRNA-PGK1 and radiotherapy. The expression of PGK1 in vivo at the mRNA and protein levels was downregulated by the treatment of shRNA1 when compared to levels following treatment with shNC and PBS after radiotherapy. The results showed that suppression of PGK1 enhanced the radiosensitivity of U251 xenografts and suggest that PGK1 may serve as a useful target in the treatment of radioresistant glioma.
\end{abstract}

\section{Introduction}

The annual incidence of gliomas which account for more than $50 \%$ of all brain tumors in adults is $6 / 100,000$ (1). Based on the histopathologic subgroups characterized by different levels of aggressiveness and malignancy, astrocytomas account for $60-70 \%$ of gliomas. Extensive surgical resection, which correlates with patient survival independently $(2,3)$, followed by

Correspondence to: Professor Hong-Yi Liu, Department of Neurosurgery, Nanjing Brain Hospital Affiliated to Nanjing Medical University, 264 Guangzhou Road, Nanjing, Jiangsu, P.R. China E-mail: hongyiliunk@163.com

Professor Hong Xiao, Department of Neuro-Psychiatric Institute, Nanjing Brain Hospital Affiliated to Nanjing Medical University, 264 Guangzhou Road, Nanjing, Jiangsu, P.R. China

E-mail: xhnkyy123@163.com

Key words: glioma, U251 xenograft, PGK1, radiosensitivity radiotherapy is one of the standard treatments for glioma. Yet, the prognosis remains dismal due to the ability of gliomas to infiltrate diffusely into the normal brain parenchyma, a direct consequence of the transformation into genetic higher-grade gliomas and recurrence (4-6). As known, radioresistance is a common phenomenon in gliomas and, to date there are no valid biomarkers for evaluating radiosensitivity.

In fact, radiotherapy which relies on the generation of oxygen super-radicals, often fails to kill tumor cells, due to inadequate oxygen stress within the tumor cell mass $(7,8)$. However, in tumor microenvironments where oxygen is scarce and glucose consumption is high, as within the expanding tumor mass, a metabolic pathway shift occurs in order to maintain local homeostasis. This phenomenon is known as the Warburg effect. Transcription of numerous enzymes such as phosphofructokinase-1 (PFK-1), lactate dehydrogenase (LDH) and glyceraldehyde-3-phosphate dehydrogenase (GAPDH) is activated in the glycolytic pathway. Among these enzymes, PGK1, an ATP-generating glycolytic enzyme, plays a vital role. It catalyzes the transfer of a high-energy phosphoryl group from the acyl phosphate of 1,3-diphosphoglycerate to ADP to produce ATP. PGK1 has also been proven to have an increased expression level in many malignant tumors (9-16). The possible role of PGK1 in radioresistance has been previously studied. Data from our laboratory showed that expression of PGK1 is significantly upregulated in radioresistant astrocytomas and radioresistant glioma $\mathrm{U} 251$ cells $(17,18)$. As an initial step toward identification of a clinically applicable method for regulating PGK1 for combination with radiotherapy, we investigated the effects of shRNA-PGK1 on the in vitro radiosensitivity of human glioma U251 cells (18). Downregulation of PGK1 by shRNA combined with radiotherapy significantly decreased cell viability and reduced the ability of migration and invasion in U251 cells. This indicates that PGK1 may be closely associated with glioma radioresponse and may be a potential target with which to sensitize conventional radiotherapy. However, the exact effect of PGK1 on the radiosensitivity in vivo using animal models has not yet been clarified. To further evaluate the potential radioresistance of PGK1, we extended these in vitro results to an in vivo xenograft model. Mice bearing U251 xenografts were exposed to shRNA-PGK1 and the level of PGK1 was determined. Radiation was then delivered in a single exposure, and shRNA-PGK1 treatment 
resulted in a significant enhancement in tumor response. These findings suggest a possible approach for the rational design of a clinical protocol combining downregulation of PGK1 and radiotherapy.

\section{Materials and methods}

Cell lines and culture. Human U251 cells were obtained from KeyGen Biotech (Nanjing, China), and radioresistant U251 cells (RR-U251 cells) were established according to a previously described method (18). The cells were cultured in Dulbecco's modified Eagle's medium (DMEM) supplemented with $10 \%$ fetal calf serum (both from Gibco-Invitrogen, Carlsbad, CA, USA), $100 \mathrm{U} / \mathrm{ml}$ of penicillin and $100 \mu \mathrm{g} / \mathrm{ml}$ of streptomycin in a $5 \% \mathrm{CO}_{2}$ humidified incubator at $37^{\circ} \mathrm{C}$.

Plasmid molecules and stable transfection in vitro. The shRNA-PGK1 plasmid and the high expression plasmid pcDNA3.1-PGK1 were synthesized commercially by GenePharma Co. Ltd. (Shanghai, China). shRNA1 (5'-GCAAG GATGTTCTGTTCTTGA-3'), shRNA2 (5'-GCTCAACAAC ATGGAGATTGG-3'), shRNA3 (5'-GGATGTCTATGTCAA TGATGC-3') and shRNA4 (5'-GAAGATTACCTTGCCTGT TGA-3') were designed to target the coding region of the human PGK1 sequence (gene ID: 5230). A negative control shRNA-NC (5'-GTTCTCCGAACGTGTCACGT-3') was also obtained from Shanghai GenePharma. U251 cells were trypsinized and harvested from a monolayer to a cell suspension in DMEM containing 10\% fetal bovine serum without antibiotics and allowed to seed overnight. When the cell confluency was 70-80\%, complexes of shRNAs or pcDNA3.1-PGK1 duplexes and Lipofectamine 2000 (Invitrogen) were prepared as follows: $4 \mu \mathrm{g}$ shRNAs or pcDNA3.1-PGK1 and $8 \mu \mathrm{l}$ of Lipofectamine 2000 were diluted in $240 \mu \mathrm{l}$ Opti-MEM I medium (Gibco) separately, and after 5 min standing at room temperature, gentle mixing was conducted. After a $20-\mathrm{min}$ incubation of this mixture, the complexes were added to each well (final concentration for DNA plasmids was $2 \mu \mathrm{g} / \mathrm{ml}$ ). The medium for transfection was replaced by complete medium at $6 \mathrm{~h}$ after transfection and observed under a fluorescence microscope (Axiovert 40 CFL; Carl Zeiss, Oberkochen, Germany) to observe the transfection efficiency at $24 \mathrm{~h}$ after transfection. The expression of PGK1 was analyzed, respectively, at 24 and $48 \mathrm{~h}$ after transfection. A control and a negative control were also placed in the 6-well plates. In addition, complete medium with G418 $(400 \mu \mathrm{g} / \mathrm{ml})$ (Keygen, Nanjing, China) was used to select the stable clones which were transfected with pcDNA3.1-PGK1.

Establishment of the nude mouse tumor xenograft models. Male Balb/c nude mice, 6-8 weeks old, purchased from the Model Animal Research Center of Nanjing University, were used in the experiments. Mice were maintained under specific pathogen-free conditions at a constant room temperature and humidity and a 12-h light/dark cycle. Sterilized food and water were provided ad libitum. Animals were subjected to an adaptation period of 2 weeks before the experiments. Transplantable U251 cells, RR-U251 cells and cells stably treated with pcDNA3.1-PGK1 in log phase growth were collected. Then, the mice received subcutaneous (s.c.) implantation of the cells $\left(3 \times 10^{7}\right)$ which were diluted in $100 \mu$ l phosphate-buffered saline (PBS) containing 50\% Matrigel (BD Biosciences, Franklin Lakes, NJ, USA) by using $0.1 \mathrm{ml} /$ inoculation in the lower back region of the mice.

Statement of ethics. Animal studies were carried out in accordance with the US National Institutes of Health Guidelines and followed the rules of the National Animal Protection of China. The present study was approved by the Medical Ethics Committee of Nanjing Brain Hospital Affiliated to Nanjing Medical University (ethic no. 2011KY001). All efforts were made to minimize suffering and to reduce the number of mice used.

Delivery of shRNA in vivo and radiotherapy. When the s.c. tumors of the nude mice were $\sim 60-80 \mathrm{~mm}^{3}$ in size, shRNAPGK1 (500 pmol/tumor, $25 \mu \mathrm{l}$ injection volume) was slowly injected into the tumors at different sites. Then, the tumors were immediately electrically pulsed (electric field strength, $180 \mathrm{~V} / \mathrm{cm}$; pulse duration, $20 \mathrm{msec}$; pulse number, 6; frequency of pulses, $1 \mathrm{~Hz}$ ) using Square Wave pulse (Ningbo Scientz Biotechnology, Ningbo, China) though 2 parallel stainless steel electrodes on each side of the long diameter of the tumor in situ. The negative control group was injected with shRNA-NC and the control group with PBS. Fifty-four nude mice were divided randomly and equally into 9 groups, and the groups were as follows: i) U251+shRNA-PGK1; ii) U251+shNC; iii) U251+PBS; iv) RR-U251+shRNA-PGK1; v) RR-U251+shNC; vi) RR-U251+PBS; vii) overexpressedPGK1 U251+shRNA-PGK1; viii) overexpressed-PGK1 U251+shNC; and viiii) overexpressed-PGK1 U251+PBS. Groups were administered the indicated treatment every $24 \mathrm{~h}$ for 14 days. After the first treatment, groups were treated with a dose of $5 \mathrm{~Gy}$ of radiation by a ${ }^{60} \mathrm{Co}$ source at $0.5 \mathrm{~Gy} / \mathrm{min}$ for 10 min every 3 days until the cumulative dose of radiotherapy reached $20 \mathrm{~Gy}$. The tumor size was measured every other day with calipers, and the tumor volume (V) was measured with all measurements in millimeters using the following formula: $\mathrm{V}=0.523 \mathrm{x}$ length $\mathrm{x}$ width ${ }^{2}$.

Real-time polymerase chain reaction ( $R T-P C R)$. Total RNA was extracted from an equal number of cells in vitro and the tumor samples with the SV Total RNA Isolation System (Promega, Madison, WI, USA) according to the manufacturer's protocol and quantified by UV absorbance spectroscopy. RNA was reverse transcribed into cDNA with a reverse transcription system (Promega). mRNA expression was determined by RT-PCR using GoTaq ${ }^{\circledR}$ qPCR Master Mix (Promega) under standard thermocycler conditions (Eppendorf AG 22331, Germany). The primers for human PGK1 were forward, 5'-ATGCTGAGGCTGTCACTCGG-3' and reverse, 5'-CACAGCAAGTGGCAGTGTCTCC-3'; and for GAPDH: forward, 5'-CGCTGAGTACGTCGTGGAGTC-3' and reverse, 5'-GCTGATGATCTTGAGGCTGTTGTC-3'. The amplification process was $95^{\circ} \mathrm{C}$ for $2 \mathrm{~min}$, then 40 cycles at $95^{\circ} \mathrm{C}$ for $45 \mathrm{sec}, 58^{\circ} \mathrm{C}$ for $45 \mathrm{sec}, 72^{\circ} \mathrm{C}$ for $45 \mathrm{sec}$, followed by $72^{\circ} \mathrm{C}$ for $10 \mathrm{~min}$. The level of PGK1 expression in the different tumor groups was presented as the theshold cycle value. The level of PGK1 mRNA was normalized against the reference gene (GAPDH). Relative gene expression values were measured 


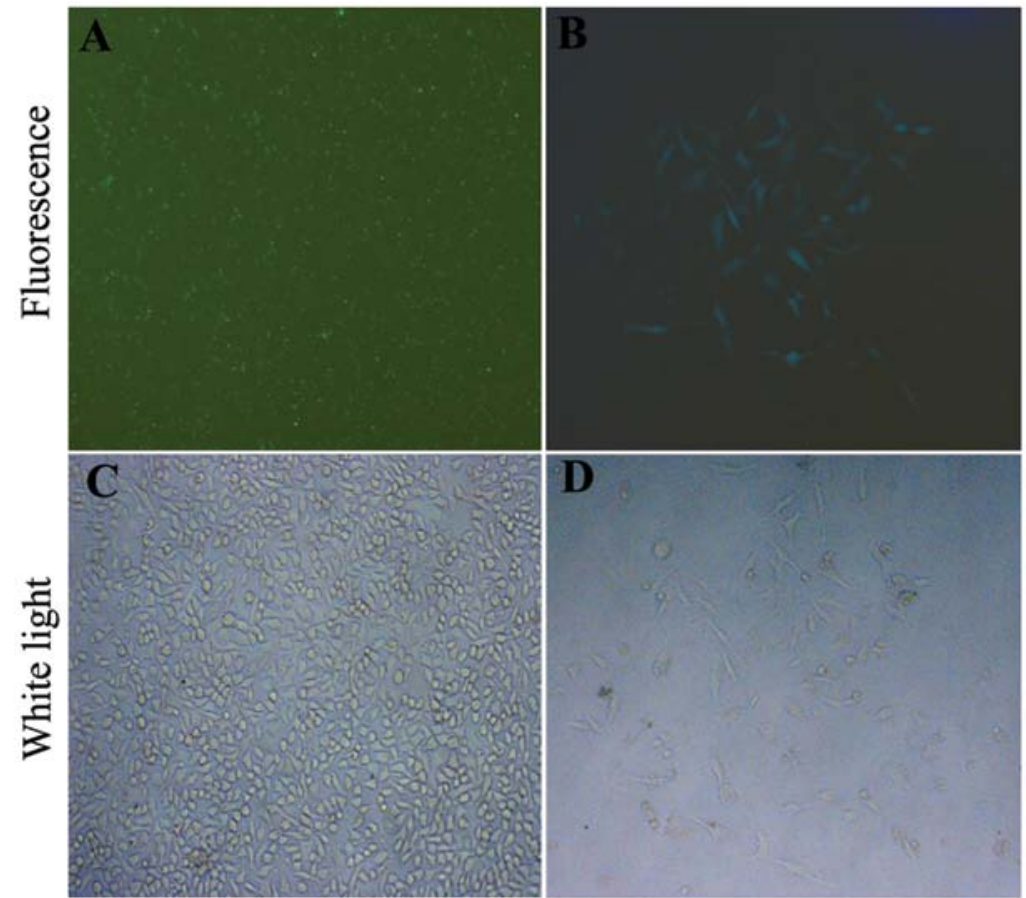

Figure 1. Establishment of PGK1-silenced U251 cells and stable overexpressed PGK1 U251 cells. (A and C) Twenty-four hours after transfection and (B and D) two weeks after the screening by G418 (400 $\mu \mathrm{g} / \mathrm{ml})$, the plates were observed under both a bright field and a fluorescence microscope system. PGK1, phosphoglycerate kinase 1 .

using the $2^{-\Delta \Delta \mathrm{Ct}}$ method as following: Ratio $=2^{-\Delta \Delta \mathrm{Ct}}$ where $\Delta \mathrm{Ct}=\mathrm{Ct}_{\mathrm{PGK} 1}-\mathrm{Ct}_{\mathrm{GAPDH}}, \Delta \Delta \mathrm{Ct}=\Delta \mathrm{Ct}_{\text {experimental group }}-\Delta \mathrm{Ct}_{\text {control group }}$

Western blot analysis. Total protein was extracted from an equal number of cells in each group and an equal weight of tumor in each group with a mixture of RIPA lysis buffer (Thermo Scientific, Runcorn UK) for $30 \mathrm{~min}$. Following centrifugation at $12,000 \times \mathrm{g}$ for $10 \mathrm{~min}$ at $4^{\circ} \mathrm{C}$, the supernatant was collected. All samples were diluted in loading buffer (Sunshine Biotechnology, Nanjing, China) and boiled for $3 \mathrm{~min}$. Total protein $(30 \mu \mathrm{g})$ was separated on $10 \%$ sodium dodecyl sulfate-polyacrylamide gel by electrophoresis (SDS-PAGE). The fractionated proteins were electro-transferred to a PVDF membrane (Sunshine Biotechnology). The membrane was blocked in 5\% skim milk and probed with antibodies from mouse against human PGK1 (Abcam, Cambridge, MA, USA) diluted in TBST buffer $(1: 1,000)$ overnight at $4^{\circ} \mathrm{C}$. Then the membrane was incubated in the goat anti-mouse horseradish peroxidase (HP)-conjugated secondary antibody $(1: 10,000)$ for $1 \mathrm{~h}$ at room temperature. Immunoreactive bands were detected using ECL chemiluminescence reagent (Thermo Scientific) and visualized by the gel image analysis software.

Immunohistochemistry. Once the cumulative radiation dose reached $20 \mathrm{~Gy}$, mice were euthanized and tumor issues were quickly excised. The tumors were fixed in neutral formalin solution and 4-mm sections were cut from each paraffin block. The sections were dewaxed in xylene and rehydrated, and an antigen retrieval step was carried out. Then, the sections were incubated in $3 \% \mathrm{H}_{2} \mathrm{O}_{2}$ in PBS for $30 \mathrm{~min}$, and blocked in PBS containing 3\% normal goat serum, $0.3 \%$ (w/v) Triton X-100 and $0.1 \%$ BSA (room temperature for $1 \mathrm{~h}$ ), followed by incubation with the primary antibody mouse against human
PGK1 $(1: 1,000)$ at $4^{\circ} \mathrm{C}$ overnight. Subsequently, sections were developed with the ABC kit and detected by DAB (both from Vector Laboratories, Burlingame, CA, USA) . The sections were then counterstained with hematoxylin, dehydrated and mounted. The images were analyzed by Image-Pro Plus.

Statistical analysis. The analysis was performed using SPSS 13.0. Statistical significance was analyzed by the Student's t-test and the Mann-Whitney U test. Data are expressed as the means \pm standard deviation (SD). $\mathrm{p}<0.05$ was considered to indicate a statistically significant difference.

\section{Results}

Overexpression of PGK1 in human glioma cells. U251 cells were stable transfected with the pcDNA3.1-PGK1 plasmid, and visible green fluorescence was observed by fluorescence microscopy after transfection (Fig. 1A and C). The efficiency was $\sim 85-90 \%$. Two weeks after the screening, most cells were killed by G418 $(400 \mu \mathrm{g} / \mathrm{ml})$; only a small number of cells survived and formed clones (Fig. 1B and D). Stable clones were selected after 3 weeks. The mRNA and protein levels of PGK1 were upregulated in the U251 cells treated with pcDNA3.1-PGK1 as determined by real-time PCR and western blotting (Fig. 2). The expression of PGK1 was significantly higher in the pcDNA3.1-PGK1 group when compared with the expression in the control group at the mRNA level $(1.29 \pm 0.08$ vs. $0.71 \pm 0.07, \mathrm{p}=0.02)$ and at the protein level $(2.28 \pm 0.09$ vs. $1.55 \pm 0.11, \mathrm{p}=0.02)$.

Selection of the most efficient shRNA specific to PGK1. Transfection of shRNA-PGK1 duplexes led to a stable exogenous gene expression with $\sim 85-90 \%$ efficiency in the U251 

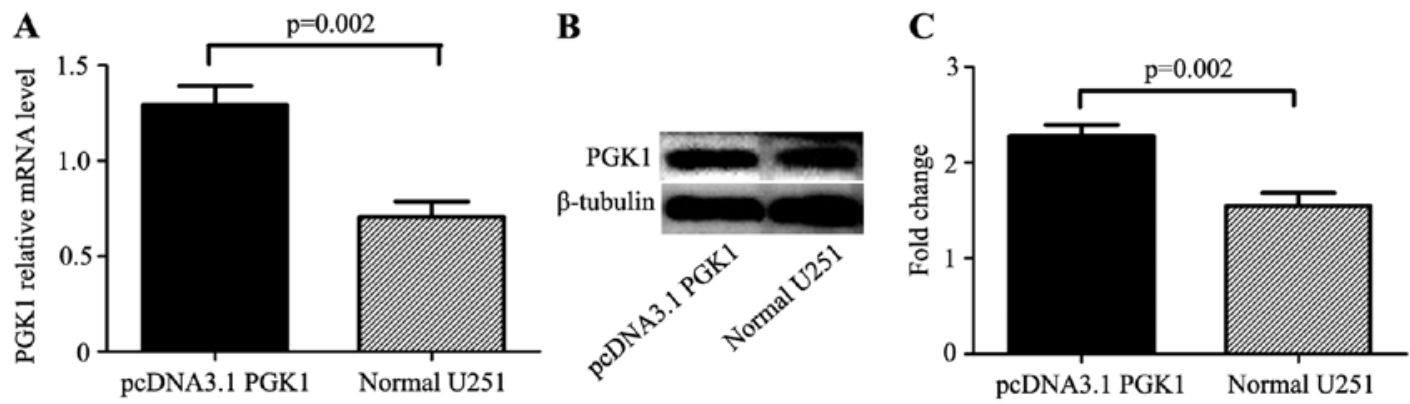

Figure 2. PGK1 expression in normal U251 and overexpressed U251 cells. (A) RT-PCR was used to detect mRNA expression of PGK1 after pcDNA3.1-PGK1 transfection. Results are presented as fold increases relative to the expression of human GAPDH. (B) Western blot analysis was used to detect the protein expression of PGK1. $\beta$-tubulin served as the loading control. (C) Relative expression of PGK1 was determined by densitometry. Data are representative of three independent experiments. PGK1, phosphoglycerate kinase 1.
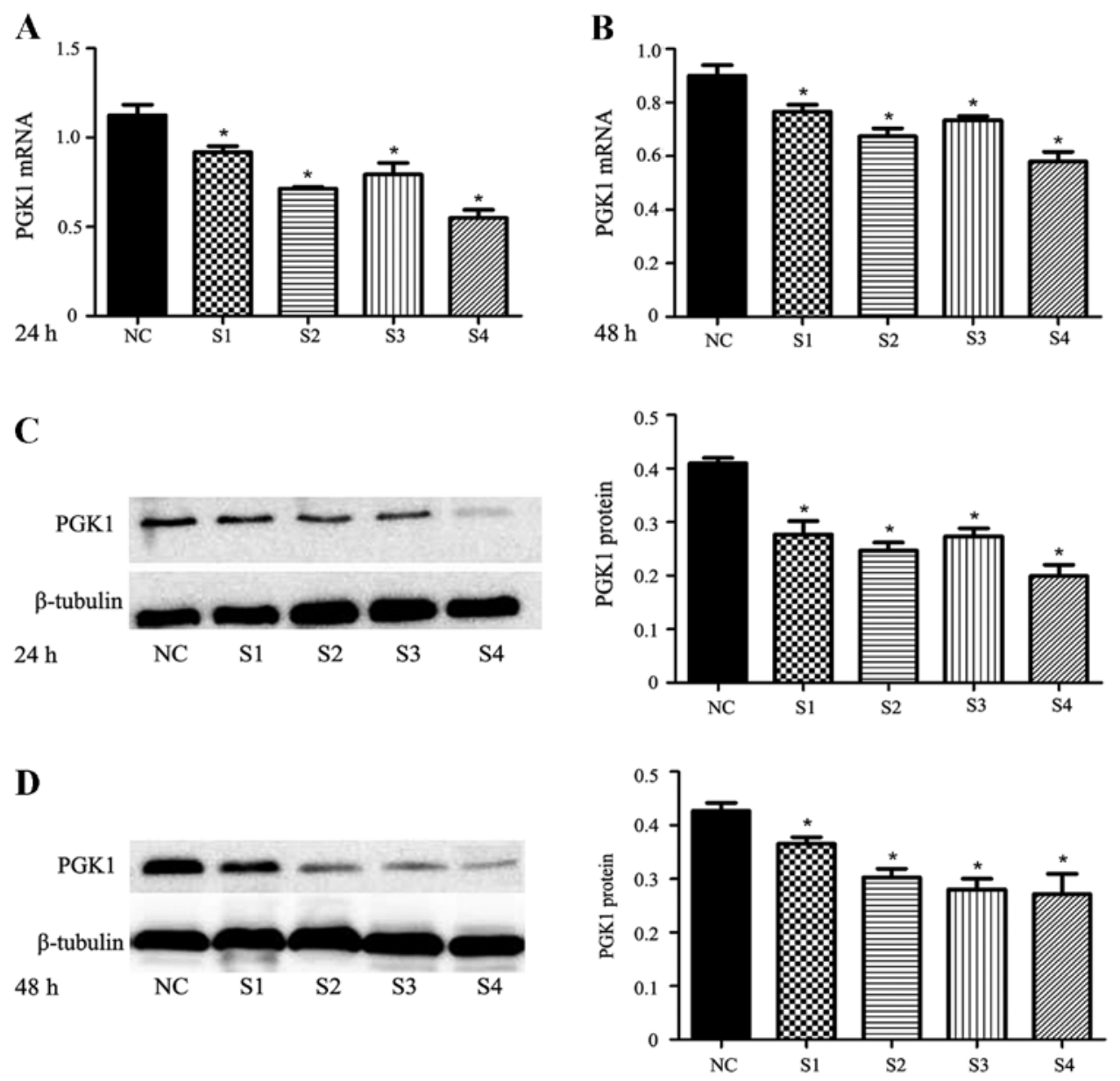

Figure 3. Selection of the most efficient shRNA and the appropriate time point. (A and B) PGK1 mRNA expression was detected at 24 and $48 \mathrm{~h}$ separately after transfection by 4 shRNAs (S1-S4) and the negative control (NC). (C and D) PGK1 protein expression was detected at 24 and $48 \mathrm{~h}$ separately after transfection by 4 shRNAs (S1-S4) and the negative control (NC). GAPDH and $\beta$-tubulin served as the loading control separately for RT-PCT and western blotting. Data are representative of three independent experiments ("p<0.05). PGK1, phosphoglycerate kinase 1.

cells; all four duplexes showed an inhibitory effect on PGK1 expression at the mRNA and protein levels (p<0.05) (Fig. 3). In order to select the most efficient shRNA and the appropriate time point at which to effectively inhibit PGK1, we further analyzed the expression of PGK1 at the mRNA and protein levels at 24 and $48 \mathrm{~h}$ after transfection. Through pairwise comparison, we found that the expression of PGK1 inhibited by shRNA4 was significantly lower than that by the other 3 series.
The inhibition ratio of PGK1 expression was $50.80 \pm 5.12 \%$ at the 24 -h time point and $35.35 \pm 5.62 \%$ at the 48 -h time point at the mRNA level $(\mathrm{p}<0.05)$. The inhibition ratio of PGK1 expression was $51.32 \pm 4.70 \%$ at the 24 -h time point and $36.56 \pm 5.48 \%$ at the 48 -h time point at the protein level $(\mathrm{p}<0.05)$. The results showed that shRNA4 was more efficient in inhibiting PGK1 expression compared with shRNA1, shRNA2 and shRNA3 at the 24-h time point after transfection. Based on the results, 
A
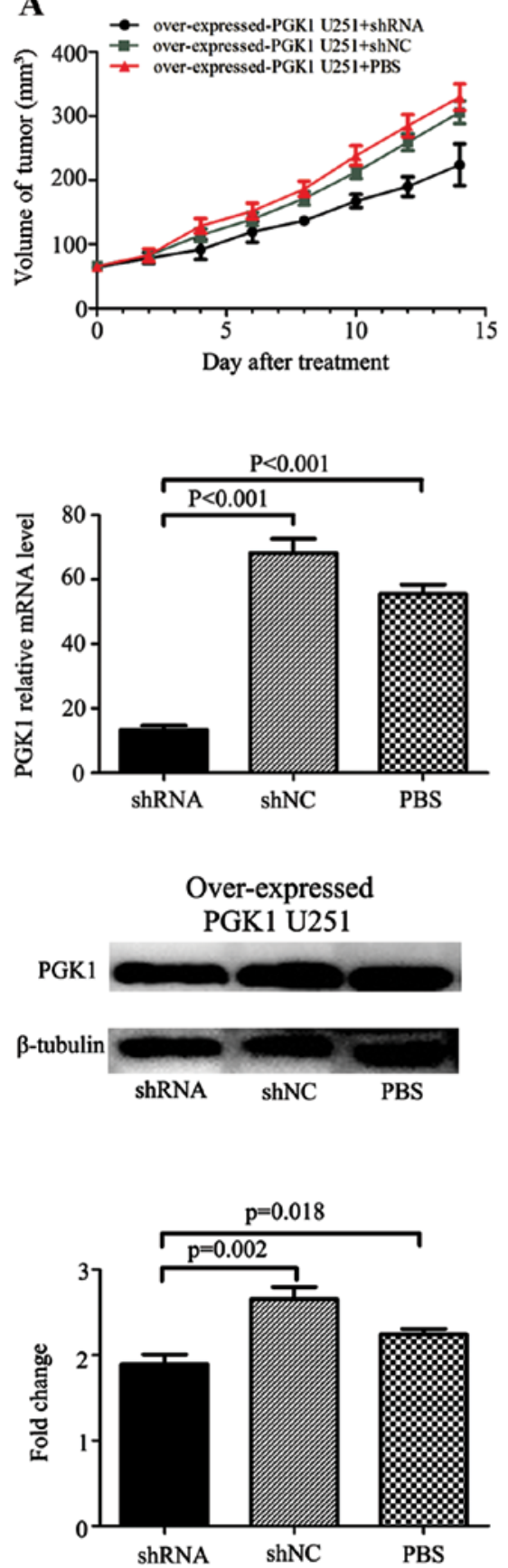

B
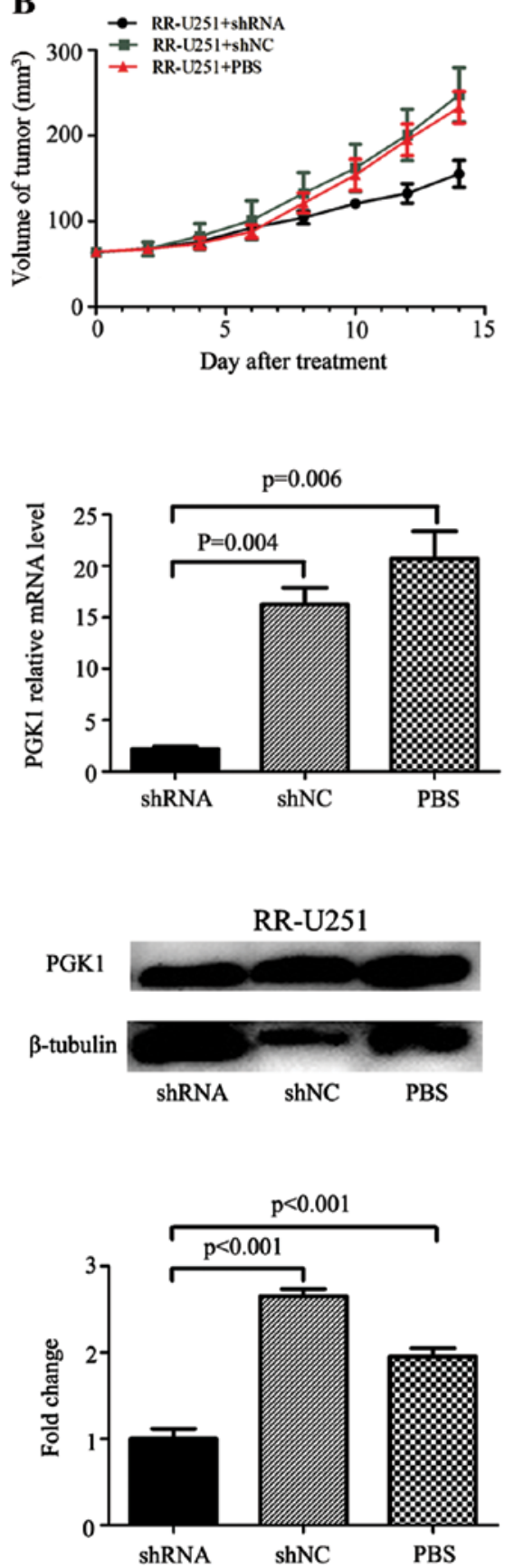

C

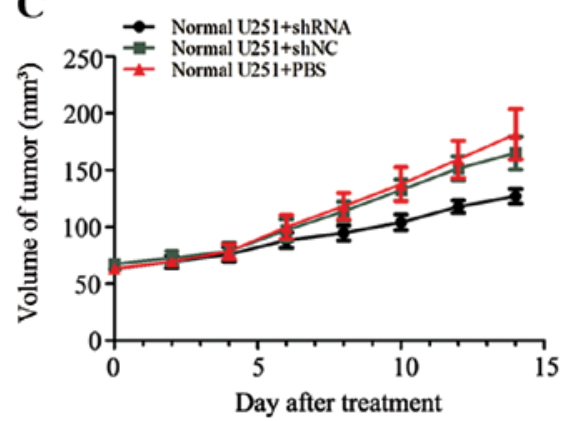

$\mathrm{P}=0.003$

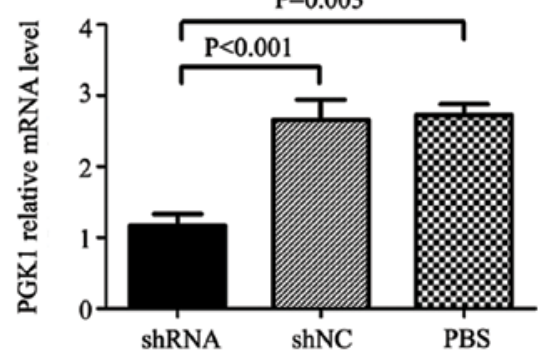

Normal U251
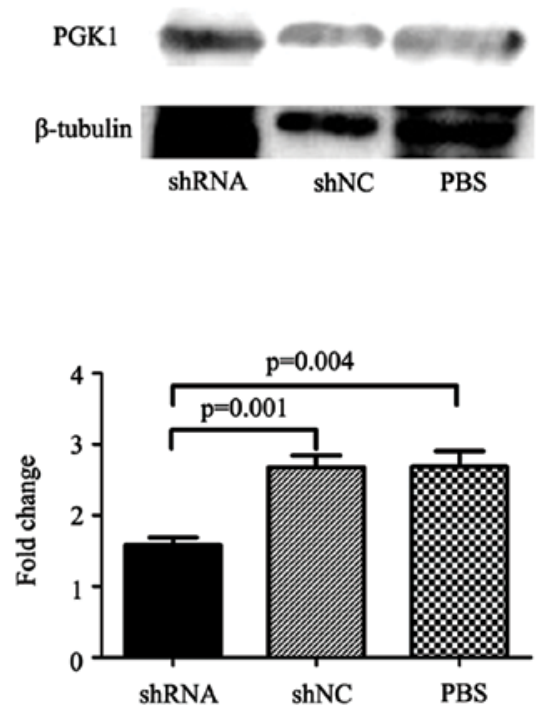

Figure 4. Analysis of radiotherapy and PGK1 in tumor xenografts. (A) Overexpressed-PGK1 U251, (B) RR-U251 and (C) normal U251 groups, for which the tumor size (mean tumor volume $\pm \mathrm{SD}$ ) was monitored every 2 over 14 days following treatment separately with shRNA, shNC or PBS. RT-PCR and western blotting were used to detect mRNA and protein expression of PGK1 in the different tumors treated with shRNA, shNC or PBS. GAPDH and $\beta$-tubulin served as the loading control separately for RT-PCT and western blotting. Data are representative of three independent experiments. PGK1, phosphoglycerate kinase 1; PBS, phosphate-buffered saline.

shRNA4 transfection for $24 \mathrm{~h}$ was used for the following experiments aimed at determining whether shRNA enhances the radioresistance of PGK1 tumor xenografts.

Combination of shRNA-PGK1 with radiotherapy in the U251 xenografts. To investigate the functional role of PGK1 in glioma growth in vivo, tumors were measured every other day. The growth of xenografts was inhibited following treatment with PGK1-shRNA in the normal U251 group, RR-U251 group and overexpressed-PGK1 U251 group. Approximately
2 days after the first treatment, overexpressed-PGK1 U251 xenografts acquired a faster growth rate compared with the normal U251 and RR-U251 group tumors (Fig. 4). At day 14 post-treatment, regarding the PBS-treated groups, the tumor size in the RR-U251 group was much larger than that in the normal U251 group $\left(232.9 \pm 18.6\right.$ vs. $181.7 \pm 22.2 \mathrm{~mm}^{3}$, $\mathrm{p}=0.002$ ), and the tumor size in the overexpressed-PGK1 U251 group was also larger than that in the normal U251 group $\left(329.6 \pm 20.6\right.$ vs. $\left.181.7 \pm 22.2 \mathrm{~mm}^{3}, \mathrm{p}=0.000\right)$. Combination of shRNA-PGK1 and radiotherapy significantly decreased the 


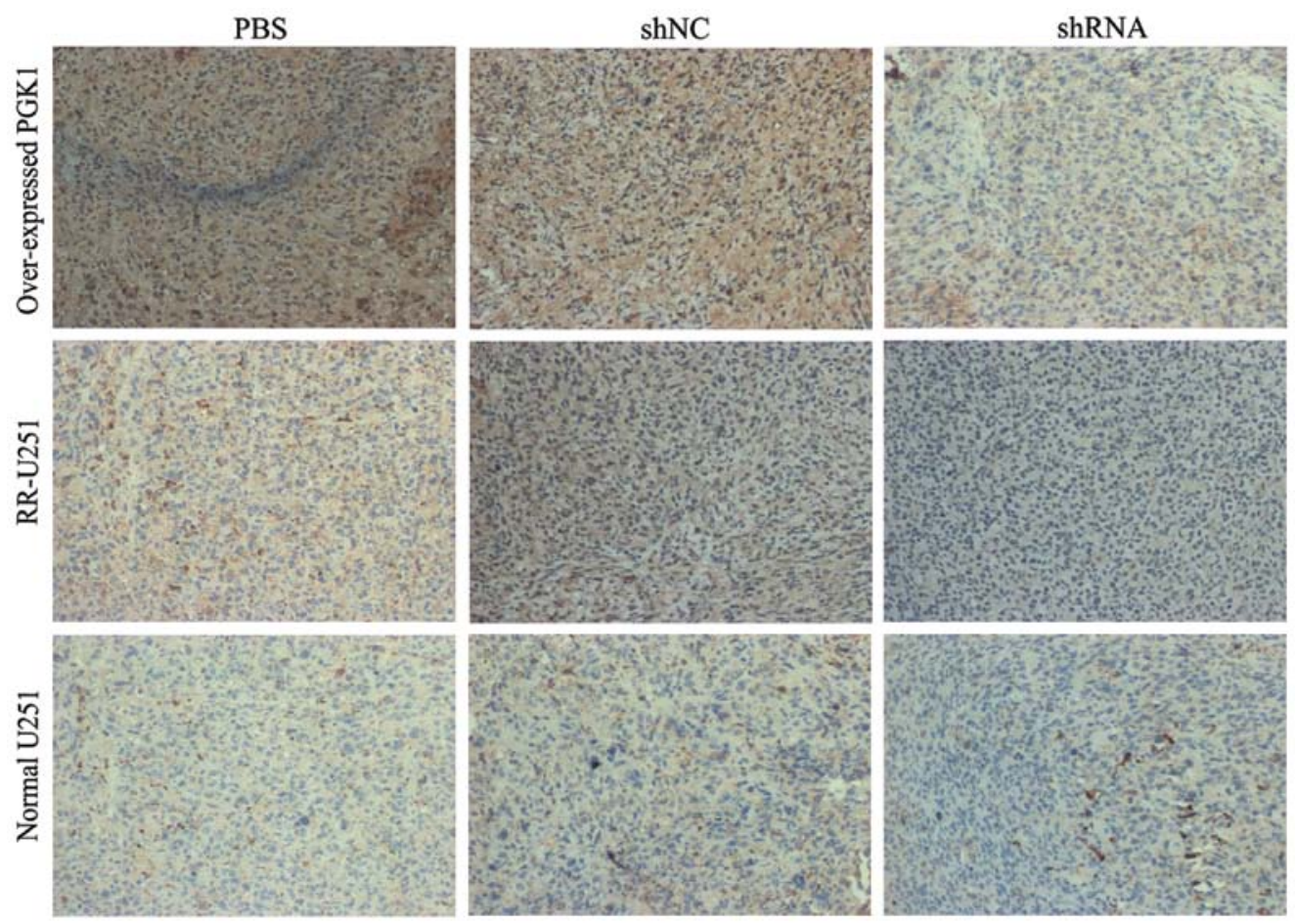

Figure 5. Detection of PGK1 in different tumors by immunohistochemistry. Sections of tumors were immunostained with the anti-PGK1 antibody. PGK1 staining (brown) is visible in clusters of tumor cells. PGK1, phosphoglycerate kinase 1.

tumor size in the normal U251 xenografts (shRNA-PGK1 group vs. shRNA-NC group: $127.2 \pm 6.0$ vs. $165.0 \pm 13.2 \mathrm{~mm}^{3}$, $\mathrm{p}=0.001$ ), and following combination with shRNA-PGK1, the tumor size also decreased comparing with the control group both in the RR-U251 xenografts (shRNA-PGK1 vs. shRNA-NC group: $155.4 \pm 14.5$ vs. $\left.247.7 \pm 29.0 \mathrm{~mm}^{3}, \mathrm{p}=0.000\right)$ and overexpressed-PGK1 U251 xenografts (shRNA-PGK1 vs. shRNA-NC group: $223.9 \pm 29.7$ vs. $\left.305.9 \pm 16.1 \mathrm{~mm}^{3}, \mathrm{p}=0.001\right)$. The data indicate that high expression of PGK1 is correlated with enhanced ability of tumor xenograft radioresistance, and shRNA-PGK1 effectively enhanced the radiosensitivity of the U251 xenografts.

Furthermore, we assessed the quantity (means \pm SD) of PGK1 expression by RT-PCR and western blotting as described in Materials and methods (Fig. 4). At the mRNA level, the data for the group treated by shRNA vs. shNC and PBS in the overexpressed-PGK1 U251 groups were respectively 13.25 \pm 1.15 , $68.21 \pm 3.60$ and $55.53 \pm 2.39$; the data for the RR-U251 groups were $2.19 \pm 0.94,16.25 \pm 1.33$ and $20.72 \pm 2.17$; the data for the normal U251 groups were $1.17 \pm 0.13,2.66 \pm 0.23$ and $2.732 \pm 0.12$ $($ all $\mathrm{p}<0.05)$. While at the protein level, the data for the group treated by shRNA vs. shNC and PBS in the overexpressedPGK1 U251 groups were respectively $1.89 \pm 0.09,2.65 \pm 0.11$ and $2.24 \pm 0.05$; the data for the RR-U251 groups were $1.00 \pm 0.09$, $2.65 \pm 0.06$ and $1.95 \pm 0.08$; the data for the normal U251 groups were $1.58 \pm 0.09,2.68 \pm 0.14$ and $2.69 \pm 0.18$ (all $p<0.05$ ). Visually, xenograft tumor sections showed extensive PGK1 staining by immunohistochemistry (Fig. 5), confirming that shRNA downregulated PGK1 expression in the xenografts in all groups. As a result, shRNA-PGK1 significantly downregulated PGK1 in the $\mathrm{U} 251$ xenografts warranting additional investigation of its therapeutic potential in glioma in the clinic.

\section{Discussion}

One important multimodal treatment of gliomas, surgical removal of the tumor followed by radiotherapy, has improved the survival of glioma patients. However, the frequency of recurrence and rapid progression in patients emphasize the need for a major enhancement of therapeutic efficacy to achieve long-term survival without relying exclusively on uncertain resistance to radiotherapy. In our experiment, we evaluated the expression of PGK1 in specimens from different models of nude mouse tumor xenografts. To our knowledge, this is the first in vivo study that has shown the relationship between PGK1 regulation by shRNA and U251 xenograft radiosensitivity. Tumors with high PGK1 expression showed higher tumorigenic properties which is consistent with our previous study (18). Notably, downregulation of the expression of PGK1 by specific small molecule inhibitors can significantly decrease the radioresistance and the progression of glioma.

It is important to note that cancer is not only a genetic disease, but is also a type of energy metabolic disease (19). Warburg proposed that the primary cause of cancer is an energy deficiency even in the presence of a high $\mathrm{O}_{2}$ concentration caused by an irreversible damage to the mitochondrial function that induces increased glycolysis which has been found in many cancers by fluorodeoxyglucose positron emission tomography $(20,21)$. Despite the universality of PET, the study of the 'bioenergetic' basis behind this phenotype has not been examined clearly, frequently or commonly by the scientific community during the past several decades. As known, hypoxia mediated by the Warburg effect is an intrinsic factor to subsequent tumor development and is a prognostic variable for unfavorable outcome, as it provides the 
mechanisms by which tumors can selectively promote a more aggressive phenotype, recruit a nutritional supply, and provide essential metabolic adaptations to ensure survival. The tumor hypoxic microenvironment can trigger the altered metabolism of the molecular basis of malignant gliomas: the glycolytic enzymes. PGK1, as a vital glycolytic enzyme, plays a vital role and catalyzes the transfer of a high-energy phosphoryl group from the acyl phosphate of 1,3-diphosphoglycerate to ADP to produce ATP. In addition to our previous study (18), Zhang et al also found that overexpression of PGK1 is related to increased necessity of energy in fast growing tumors due to protein synthesis and degradation pathways (22). In the microenvironment, hypoxia augments the expression of key mediators, such as HIF-1, c-myc and MYCN, which have been related to PGK1 and resistance to radiotherapy (23-26). These factors can hasten the expression and the activity of PGK1. For example, PGK1 was induced by one of the above factors, HIF-1, a key transcription factor involved in glycolytic energy metabolism; its expression is correlated with treatment resistance and overall poor prognosis. HIF-1 can also promote the production of vascular endothelial growth factor (VEGF) which in turn stimulates both angiogenesis and glycolytic enzyme activity with the ability to facilitate anaerobic production of ATP (26). Notably, a recent study also demonstrated that HIF-1 is not only induced by hypoxia, but also by glycolytic metabolites (27). Thus, we hypothesized that the combinatorial effects of PGK1 and HIF-1 contribute to the poor sensitivity to radiotherapy. Downstream targets of PGK1, E-cadherin and $\beta$-catenin (13), are reportedly associated with tumorigenic properties, including cell viability, migration, invasion and angiogenesis ability which further promote tumor radioresistance. This is consistent with our data of U251 xenografts showing that PGK1 is essential for increased tumor size and significant resistance to radiotherapy.

Furthermore, the accumulation of lactate which is the final product of glycolysis, increased by PGK1, may also facilitate radioresistance. Lactate production may be the result of a compensatory increase in the constitutive level of expression of glycolytic enzymes, leading to a high rate of glycolytic capacity (28). Lactate is expected to lead to acidosis of the cell culture medium during hypoxia, resulting in glioma radioresistance. The hypothesis is consistent with a study by Hsu and Sabatini (29) who found that glycolysis pathway enzymes have an antagonized apoptotic effect which can lead to the tolerance to radiotherapy on apoptosis of malignant tumors. Moreover, lactic acid enables the tumor in an acidic tumor microenvironment to favor glioma invasion obtaining tumor immune escape by inhibiting lymphocyte function compared with the edge of glioma cells which can have relatively high oxygen content and make use of lactic acid for oxidative phosphorylation.

Ionizing radiation results in tumor DNA damage, mainly by double-strand breaks, making them unable to divide and grow (30). As a result of the glycolysis of PGK1, it further affects DNA replication responses and the repair system (31-33). The present study indicated that overexpression of PGK1 led to glioma radioresistance, which may also be due to the DNA damage repair ability of PGK1 and can be a serious hurdle to diminish susceptibility of the irradiated glioma cells to undergo apoptosis. This finally leads to the occurrence of tumor radioresistance and recurrence. Yet, addi- tional studies are needed to confirm this hypothesis with cell cycle checkpoint pathways which have been proven to play an important role in the development of radioresistance in tumor cells $(34,35)$.

Overall, our results revealed that PGK1 is sufficient to promote radioresistance, and appropriate shRNAs can significantly downregulate PGK1 in U251 xenografts. This confirms our previous study that PGK1 can enhance radioresistance in clinical glioma and U251 cells in vitro. However, future investigation into the regulation of the enzymatic activity of PGK1 in additional tumor cell lines is critical. Among these tumor cell lines, neural stem cells (NSCs) have attracted more and more attention. The cells can act though the preferential activation of the DNA damage response, induction of cell cycle arrest and then repair of damaged DNA to improve radiation resistance (36). Zieker et al (37) initially explained the relationship between PGK1 and stem cells in gastric cancer. Ultimately, based on a better understanding of the role of PGK1 in glioma progression and post-operative resistance, pharmacological agents designed as agonists of the silencing of PGK1 may slow tumor growth or may convert a therapeutically resistant tumor to one that is sensitive to treatment.

\section{Acknowledgements}

The present study was supported by the grant NSFC81172390 from the National Science Foundation of China, and the grant ZKX10021 from the Health Bureau of Nanjing.

\section{References}

1. De Witt Hamer PC, Robles SG, Zwinderman A, Duffau H and Berger M: Impact of intraoperative stimulation brain mapping on glioma surgery outcome: a meta-analysis. J Clin Oncol 30: 2559-2565, 2012.

2. Smith JS, Chang EF, Lamborn KR, et al: Role of extent of resection in the long-term outcome of low-grade hemispheric gliomas. J Clin Oncol 26: 1338-1345, 2008.

3. Sanai N and Berger MS: Glioma extent of resection and its impact on patient outcome. Neurosurgery 6: 753-764, 2008.

4. Wen PY and Kesari S: Malignant gliomas in adults. N Engl J Med 359: 492-507, 2008.

5. Benjamin R, Capparella J and Brown A: Classification of glioblastoma multiforme in adults by molecular genetics. Cancer $\mathrm{J} 9$ : 82-90, 2003.

6. Behin A, Hoang-Xuan K, Carpentier AF and Delattre JY: Primary brain tumours in adults. Lancet 361: 323-331, 2003.

7. Höckel M, Schlenger K, Mitze M, Schäffer U and Vaupel P: Hypoxia and radiation response in human tumors. Semin Radiat Oncol 6: 3-9, 1996.

8. Unruh A, Ressel A, Mohamed HG, et al: The hypoxia-inducible factor- $1 \alpha$ is a negative factor for tumor therapy. Oncogene 22 : 3213-3220, 2003.

9. Ahmad SS, Glatzle J, Bajaeifer K, et al: Phosphoglycerate kinase 1 as a promoter of metastasis in colon cancer. Int $\mathbf{J}$ Oncol 43: 586-590, 2013.

10. Chen G, Gharib TG, Wang H, et al: Protein profiles associated with survival in lung adenocarcinoma. Proc Natl Acad Sci USA 100: 13537-13542, 2003.

11. Zieker D, Königsrainer I, Tritschler I, et al: Phosphoglycerate kinase 1 a promoting enzyme for peritoneal dissemination in gastric cancer. Int J Cancer 126: 1513-1520, 2010.

12. Wang J, Ying G, Wang J, et al: Characterization of phosphoglycerate kinase-1 expression of stromal cells derived from tumor microenvironment in prostate cancer progression. Cancer Res 70: 471-480, 2010.

13. Wang J, Wang J, Dai J, et al: A glycolytic mechanism regulating an angiogenic switch in prostate cancer. Cancer Res 67: 149-159, 2007. 
14. Cecconi D, Palmieri $M$ and Donadelli $M$ : Proteomics in pancreatic cancer research. Proteomics 11: 816-828, 2011

15. Lincet H, Guével B, Pineau C, et al: Comparative 2D-DIGE proteomic analysis of ovarian carcinoma cells: toward a reorientation of biosynthesis pathways associated with acquired platinum resistance. J Proteomics 75: 1157-1169, 2012.

16. Duan Z, Lamendola DE, Yusuf RZ, Penson RT, Preffer FI and Seiden MV: Overexpression of human phosphoglycerate kinase 1 (PGK1) induces a multidrug resistance phenotype. Anticancer Res 22: 1933-1941, 2002.

17. Yan H, Yang K, Xiao H, Zou YJ, Zhang WB and Liu HY: Over-expression of cofilin-1 and phosphoglycerate kinase 1 in astrocytomas involved in pathogenesis of radioresistance. CNS Neurosci Ther 18: 729-736, 2012.

18. Ding H, Cheng YJ, Yan H, et al: Phosphoglycerate kinase 1 promotes radioresistance in U251 human glioma cells. Oncol Rep 31: 894-900, 2014.

19. Wu W and Zhao S: Metabolic changes in cancer: beyond the Warburg effect. Acta Biochim Biophys Sin 45: 18-26, 2013.

20. Gatenby RA and Gillies RJ: Why do cancers have high aerobic glycolysis? Nat Rev Cancer 4: 891-899, 2004.

21. Seemann MD: PET/CT: fundamental principles. Eur J Med Res 9: 241-246, 2004.

22. Zhang DH, Tai LK, Wong LL, Chiu LL, Sethi SK and Koay ES Proteomic study reveals that proteins involved in metabolic and detoxification pathways are highly expressed in HER-2/neupositive breast cancer. Mol Cell Proteomics 4: 1686-1696, 2005.

23. Luo FM, Liu XJ, Yan NH, et al: Hypoxia-inducible transcription factor- $1 \alpha$ promotes hypoxia-induced A549 apoptosis via a mechanism that involves the glycolysis pathway. BMC Cancer 6: 26, 2006.

24. Qing G, Skuli N, Mayes PA, et al: Combinatorial regulation of neuroblastoma tumor progression by $\mathrm{N}-\mathrm{Myc}$ and hypoxia inducible factor HIF-1 $\alpha$. Cancer Res 70: 10351-10361, 2010

25. Aebersold DM, Burri P, Beer KT, et al: Expression of hypoxiainducible factor-1 $\alpha$ : a novel predictive and prognostic parameter in the radiotherapy of oropharyngeal cancer. Cancer Res 61: 2911-2916, 2001
26. Ryan HE, Lo J and Johnson RS: HIF-1 alpha is required for solid tumor formation and embryonic vascularization. EMBO J 17: 3005-3015, 1998

27. Lu H, Forbes RA and Verma A: Hypoxia-inducible factor 1 activation by aerobic glycolysis implicates the Warburg effect in carcinogenesis. J Biol Chem 277: 23111-23115, 2002.

28. Rodríguez-Enríquez S and Moreno-Sánchez R: Intermediary metabolism of fast-growth tumor cells. Arch Med Res 29: 1-12, 1998.

29. Hsu PP and Sabatini DM: Cancer cell metabolism: Warburg and beyond. Cell 134: 703-707, 2008.

30. Ward JF: Radiation mutagenesis: the initial DNA lesions responsible. Radiat Res 142: 362-368, 1995.

31. VandeBerg JL: The phosphoglycerate kinase isozyme system in mammals: biochemical, genetic, developmental, and evolutionary aspects. Isozymes Curr Top Biol Med Res 12: 133-187, 1985.

32. Popanda O, Fox G and Thielmann HW: Modulation of DNA polymerases $\alpha, \delta$ and $\varepsilon$ by lactate dehydrogenase and 3-phosphoglycerate kinase. Biochim Biophys Acta 1397: 102-117, 1998.

33. Vishwanatha JK, Jindal HK and Davis RG: The role of primer recognition proteins in DNA replication: association with nuclear matrix in HeLa cells. J Cell Sci 101: 25-34, 1992.

34. McCord AM, Jamal M, Williams ES, Camphausen K and Tofilon PJ: CD133+ glioblastoma stem-like cells are radiosensitive with a defective DNA damage response compared with established cell lines. Clin Cancer Res 15: 5145-5153, 2009.

35. Narayana A, Gruber D, Kunnakkat S, et al: A clinical trial of bevacizumab, temozolomide, and radiation for newly diagnosed glioblastoma. J Neurosurg 116: 341-345, 2012.

36. Bao S, Wu Q, McLendon RE, et al: Glioma stem cells promote radioresistance by preferential activation of the DNA damage response. Nature 444: 756-760, 2006.

37. Zieker D, Bühler S, Ustündag Z, et al: Induction of tumor stem cell differentiation - novel strategy to overcome therapy resistance in gastric cancer. Langenbecks Arch Surg 398: 603-608, 2013. 